\title{
Generic properties of periodic reflecting rays
}

\author{
LUCHEZAR STOJANOV \\ Institute of Mathematıcs, Bulgarian Academy of Sciences, 1090 Sofia, Bulgaria
}

(Recelved 3 December 1985, revised 7 Aprll 1986 and 7 July 1986)

Abstract It is shown that for generic domains $D$ in $\mathbb{R}^{n}, n \geq 2$, every periodic billiard trajectory in $D$ passes only once through each of its reflection points, and any two different periodic billiard trajectones in $D$ have no common reflection point

\section{Introduction}

Given a compact domain $D$ in $\mathbb{R}^{n}, n \geq 2$, with smooth boundary $X=\partial D$, we consider periodic billard trajectories in $D$, and call them periodic reflecting rays on $X$ Generic properties of smooth compact $(n-1)$-dimensional submanifolds $X$ of $\mathbb{R}^{n}$ concernıng perıodic reflectıng rays on them were establıshed by Lazutkın [5] and Petkov and Stojanov [9], [10], [11] (see also [7] and [8]) In [5] Lazutkın proved an analogue of Kupka-Smale theorem for billiards in strictly convex domains in $\mathbb{R}^{2}$ Studying inverse spectral problems in connection with the so-called Poisson relation for manifolds with boundary ( $c f[2],[4],[6]), \mathrm{V}$ Petkov and the present author proved that generically most $X$ in $\mathbb{R}^{n}$ have the following properties

(1) every two different periodic reflecting rays on $X$ have rationally independent lengths ([9]),

(11) (for $n=2$ ) there do not exist generalized periodic geodesics on $X$ ([9], [10]),

(111) for every $s \geq 2$ there are at most a finite number of periodic reflectıng rays on $X$ having exactly $s$ reflection points ([11]),

(1v) there do not exist periodic reflectıng rays on $X$ having segments tangent to $X([10],[11])$,

(v) the spectrum of the Poincare map related to every periodic reflectıng ray on $X$ does not contain roots of unity ([11])

In [9], [10] we had to overcome among others the following two difficulties

(A) in general a periodic reflectıng ray could pass two or more tımes (in different directions) through some of its reflection points (see figure 1),

(B) some different periodic reflecting rays could have common reflection points (see figure 2)

In this paper we prove that for generic $X$ in $\mathbb{R}^{n}$ the phenomena (A) and (B) cannot occur The latter means that for generic $X$ given $x \in X$, there exist at most two directions $v \neq 0$ in $\mathbb{R}^{n}$ which are symmetric with respect to the normal $N_{x}$ to $X$ at $x$ (and of course may coincide with $N_{x}$ ) such that starting from $x$ in the direction $v$ and reflecting on $X$ satisfying the usual law of reflection, we get a periodic 
reflectıng ray on $X$ Note that, according to (111), for generic $X$ there are at most a countable number of such $x \in X$ for which there exist directions $v$ with the above properties

The proofs of our results use the technique of [9], [10] which is based on the mult1-jet transversality theorem One of these results (see theorem $A$ in $\S 2$ ) has been already used to prove the main theorem in [11], and both theorems A and B established below could be used to simplify the proofs in [9], [10] and to get other properties of genenc $X$ in $\mathbb{R}^{n}$

Thanks are due to the referee for his remarks and suggestions Especially, he pointed out that theorem B (the proof of which in the first version of the paper was different from the present one) can be established using the same idea as those in the proof of theorem $A$

\section{Definitions and main results}

Let $X$ be a smooth compact $(n-1)$-dimensional submanifold of $\mathbb{R}^{n}, n \geq 2$, and $C^{\infty}\left(X, \mathbb{R}^{n}\right)$ be the set of all smooth maps $X \rightarrow \mathbb{R}^{n}$ endowed with the Whitney $C^{\infty}$ topology (cf ch II of [1] or 21 of [3]) Denote by $C_{\mathrm{emb}}^{\infty}\left(X, \mathbb{R}^{n}\right)$ the subspace of $C^{\infty}\left(X, \mathbb{R}^{n}\right)$ consistıng of all smooth immersive embeddings $X \rightarrow \mathbb{R}^{n}$ As is well known, $C^{\infty}\left(X, \mathbb{R}^{n}\right)$ is a Baire space and $C_{\mathrm{emb}}^{\infty}\left(X, \mathbb{R}^{n}\right)$ is open in it (cf loc cit), therefore the latter is also a Baire space

By a periodic reflecting ray on $X$ we mean a closed curve $\gamma$ formed by a finite number of straightline segments $l_{1}, l_{2}, \quad, l_{k}$, where $l_{l}=\left[x_{1}, x_{i+1}\right]=\left\{z \in \mathbb{R}^{n} z=p x_{1}+\right.$ $\left.(1-p) x_{i+1}, 0 \leq p \leq 1\right\}, x_{i} \in X(l=1,2, \quad, k), x_{k+1}=x_{1}$, such that any open segment $i_{l}=\left(x_{1}, x_{1+1}\right)$ does not intersect transversally $X$, and for every $\imath=1, \quad, k, l_{1}$ and $l_{\imath+1}$ make equal acute angles with one of the normal vectors $N_{i+1}$ to $X$ at $x_{1+1}$ (for convenience we set $l_{k+1}=l_{1}$ ), and $l_{i}, l_{t+1}$ and $N_{t+1}$ lie in a common two-dimensional plane The points $x_{1}, x_{2}, \quad x_{k}$ are called reflection points of $\gamma$

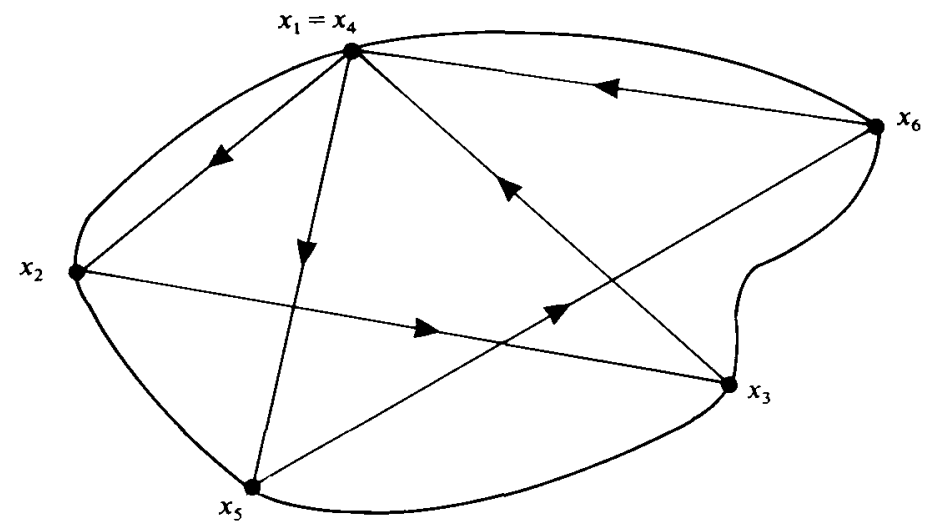

FIGURE 1

It may happen that some segments $l_{\imath}$ of $\gamma$ are tangent to $X$ at some interior point $z$ of $l_{\text {i }}$ (see figure 3) Note especially that points like $z_{1}$ on figure 3 are not considered as reflection ones, while $z_{2}$ is a reflection point Clearly, a periodic reflecting ray on 
$X$ may lie 'inside $X$ ' as well as 'outside $X$ ' (see figure 4) Moreover, some of its reflection points may coincide (see figure 1) We mention also that $X$ is not assumed to be connected

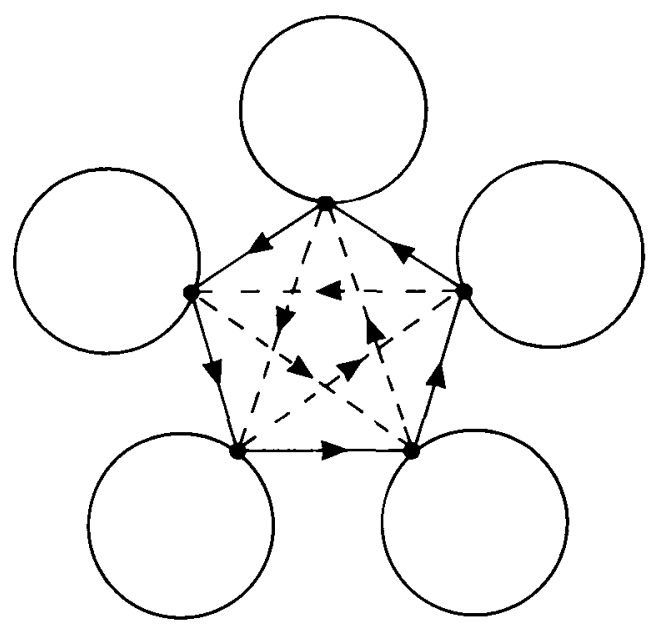

FIGURE 2

If there is a segment $l_{t}=\left[x_{t}, x_{t+1}\right]$ of $\gamma$ which is orthogonal to $X$ at $x_{t}$ or $x_{t+1}$, then $\gamma$ is called a symmetric ray ([5]) Otherwise, $\gamma$ will be called a non-symmetric ray In the first case either $k=2$ or $k>2$ and exactly two segments of $\gamma$ are orthogonal to $X$ at some of their end points (see figure 4)

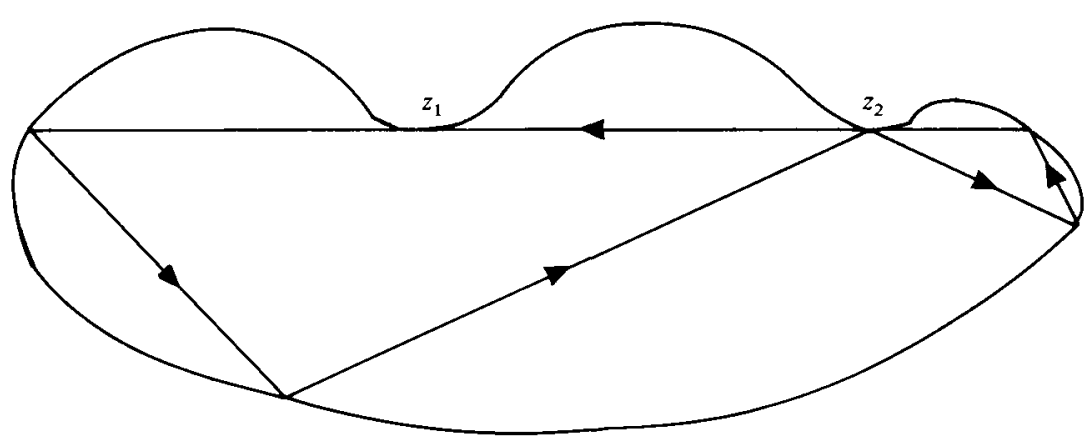

FigURE 3

Given a periodic reflectıng ray $\gamma$ denote by $m$ the number of all different segments of $\gamma$ (thus for symmetric $\gamma$ we should take only a half of the segments of $\gamma$ ), and let $s$ be the number of all different reflection points of $\gamma$ The number $d(\gamma)$, defined by $d(\gamma)=m-s$ for non-symmetric $\gamma$ and by $d(\gamma)=m+1-s$ for symmetric $\gamma$, will be called the defect of $\gamma$ 


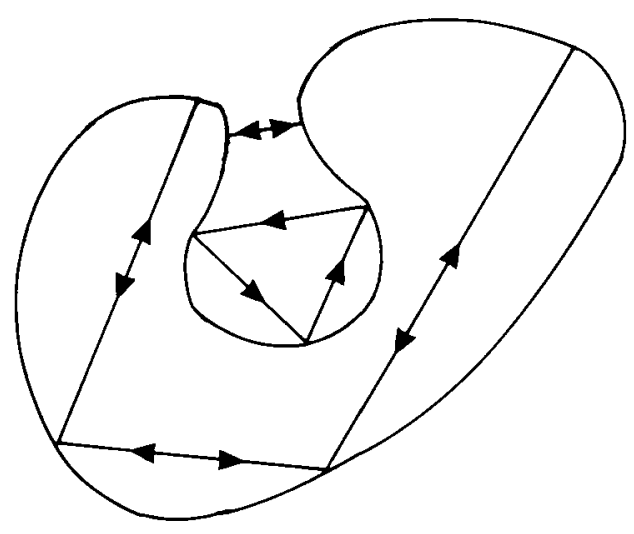

FiguRE 4

We are ready to state our results

THEOREM A Let $X$ be a smooth compact ( $n-1)$-dimenstonal submanifold of $\mathbb{R}^{n}$, $n \geq 2$, and $\mathscr{A}$ be the set of those $f \in C_{\mathrm{emb}}^{\infty}\left(X, \mathbb{R}^{n}\right)$ such that every periodic reflecting rav on $f(X)$ has zero defect Then $\mathscr{A}$ contains a residual subset of $C_{\mathrm{emb}}^{\infty}\left(X, \mathbb{R}^{n}\right)$

Usıng different methods Lazutkın [5] proved that for generıc strictly convex $X$ in $\mathbb{R}^{2}$ the inequality $d(\gamma) \leq 2$ is fulfilled for every periodic reflectıng ray $\gamma$ on $X$

THEOREM B Let $X$ be as in theorem $A$, and $\mathscr{B}$ be the set of those $f \in C_{\mathrm{emb}}^{x}\left(X, \mathbb{R}^{n}\right)$ such that any two different periodic reflecting ravs on $f(X)$ have no common reflection point Then $\mathscr{B}$ contains a residual subset of $C_{\mathrm{emb}}^{\infty}\left(X, \mathbb{R}^{n}\right)$

Roughly speaking the idea of the proof of theorem $\mathrm{A}$ is the following Let $\gamma$ be a periodic reflecting ray on $X$ with $s$ different reflection points $x_{1}, x_{2}, \quad, x$, Then $\gamma$ passes through the points $x_{i}$ in a certain pattern For fixed $s$ the number of all those patterns is finite, so we can restrict our considerations to periodic reflecting rays $\gamma$ having $s$ different reflection points ( $s$ being fixed) which are visited by $\gamma$ in a fixed pattern Every such ray is determined by the points $x_{i}$, that is by $s(n-1)$ unknowns since $\operatorname{dim} X=n-1$ On the other hand, any two successive segments of $y$ determine lines which are symmetric with respect to the normal to $X$ at their common end $x_{i}$, and this gives $n-1$ conditions for the points $x_{1}, \quad, x_{s}$ Therefore, if $\gamma$ passes only once through each of its reflection points, then the general number of the conditions is $s(n-1)$ In the other case we have more conditions than unknowns Applying transversality type arguments we see that for generic $X$ this system has no solution

The same idea can be exploited to prove theorem B Now for fixed $s$ we consider pairs $(\gamma, \delta)$ of periodic reflecting rays on $X$ having together exactly $s$ different reflection points, and such that any of the rays $\gamma, \delta$ passes in a certain fixed pattern through its reflection points Again the number of the unknowns is $s(n-1)$ and, if $\gamma$ and $\delta$ have at least one common reflection point, then we get more than $s(n-1)$ 
conditions which are of the same type as those considered above This enables us to use the arguments from the proof of theorem $A$

Throughout the paper smooth means $C^{\infty}$, although everything is true replacing $C^{\infty}$ with $C^{k}$ for $k=1,2, \quad$ Note also that our results remain true for non-compact $X$, if we consider only such periodic reflecting rays $\gamma$ on $X$ that for every reflection point $x$ of $\gamma$ all segments of $\gamma$ passing through $x$ make acute angles with one and the same normal vector to $X$ at $x$

\section{Preltminaries}

Here we give a construction from [9] which describes analytically the periodic reflecting rays and provides a simple classification of them

31 We use $\langle$,$\rangle to denote the standard inner product in \mathbb{R}^{n}$, and by \|\| the induced norm in $\mathbb{R}^{n}$ Given a map $g \quad Y \rightarrow Z$ and an integer $s>0$, the product $g^{s} Y^{s} \rightarrow Z^{s}$ is defined by $g^{s}\left(y_{1}, \quad, y_{s}\right)=\left(g\left(y_{1}\right), \quad, g\left(y_{s}\right)\right)$ For every set $A$ we put

$$
A^{(s)}=\left\{\left(a_{1}, \quad, a_{s}\right) \in A^{s} \quad a_{t} \neq a_{j} \quad \text { whenever } \imath \neq j\right\}
$$

32 Let $k \geq s \geq 2$ be integers and

be a map with

$$
\omega\{1,2, \quad, k\} \rightarrow\{1,2, \quad, s\}
$$

$$
\omega(l) \neq \omega(l+1) \quad(l=1,2, \quad, k)
$$

We set for convenience $\omega(p)=\omega(\imath)$ for $p=l+m k, l=1,2, \quad, k, m$ an integer If we have

$$
\{\omega(\iota), \omega(l+1)\} \neq\{\omega(\jmath), \omega(\jmath+1)\}
$$

whenever $1 \leq l<J \leq k$, then $\omega$ will be called a non-symmetric map If $k=2 m$ and there is $\iota_{0}=1,2, \quad, k$ such that (3 3) holds for $\iota_{0} \leq l<J \leq \imath_{0}+m$, and

$$
\omega\left(l_{0}+m+\jmath\right)=\omega\left(\iota_{0}+m-\jmath\right) \quad(\jmath=1,2, \quad, m-1),
$$

then the map $\omega$ will be called symmetric By admissible map we mean a map (31) with (3 2) which is either symmetric or non-symmetric

(3 3) Let (3 1) be an admissible map Define

$$
I_{1}=I_{i}(\omega)=\{j \text { there is } t=1, \quad, k \text { with }\{l, J\}=\{\omega(t), \omega(t+1)\}\}
$$

for $\imath \in \operatorname{Im} \omega$, and

$$
U_{\omega}=\left\{\left(y_{1}, \quad, y_{s}\right) \in\left(\mathbb{R}^{n}\right)^{(s)} y_{i} \notin \text { convex hull }\left\{y_{J} j \in I_{1}\right\}, l \in \operatorname{Im} \omega\right\}
$$

Clearly, $U_{\omega}$ is an open subset of $\left(\mathbb{R}^{n}\right)^{(s)}$ Define the map $F\left(\mathbb{R}^{n}\right)^{(s)} \rightarrow \mathbb{R}$ by

$$
F\left(y_{1}, \quad, y_{s}\right)=\sum_{i=1}^{k}\left\|y_{\omega(1)}-y_{\omega(1+1)}\right\|
$$

Suppose $Y$ is a smooth compact $(n-1)$-dimensional submanıfold of $\mathbb{R}^{n}$ A periodic reflecting ray $\gamma$ on $Y$ will be said to be of type $\omega$ if there exist $s$ different points $y_{1}, \quad, y_{s}$ of $Y$ so that

$$
y_{\omega(1)}, y_{\omega(2)}, \quad, y_{\omega(k)}, y_{\omega(k+1)}=y_{\omega(1)}
$$

are all successive reflection points of $\gamma$ In this case we have $y=\left(y_{1}, \quad, y_{s}\right) \in U_{\omega}$, 
and the length of $\gamma$ is equal to $F(y)$ Note also that $I_{i}(\omega)$ is just the set of those $J$ such that there is a segment of $\gamma$ joinıng $y_{l}$ and $y_{j}$

If $Y=f(X)$ for some $f \in C_{\mathrm{emb}}^{\infty}\left(X, \mathbb{R}^{n}\right)$, then there exists an element $x=\left(x_{1}, \quad, x_{\mathrm{s}}\right)$ of $X^{(s)}$ with $f\left(x_{\imath}\right)=y_{\imath}(l=1, \quad, s)$ Clearly $y=f^{s}(x) \in U_{\omega}$ and $x$ is a critical point of $F \circ f^{s}$

34 Lemma ([9]) For every $y \in U_{\omega}$ and every $t \in \operatorname{Im} \omega$ there exists $t=1,2, \quad, n$ with $\left(\partial F / \partial y_{t}^{(t)}\right)(y) \neq 0\left(y_{t}=\left(y_{i}^{(1)}, \quad, y_{i}^{(n)}\right) \in \mathbb{R}^{n}\right)$

(3 5) Fix an admissible map (3 1) and consider the s-fold bundle of the 1-jets $J_{s}^{1}\left(X, \mathbb{R}^{n}\right)(\operatorname{cf}[1, \mathrm{p} 57])$ For every $f \in C^{\infty}\left(X, \mathbb{R}^{n}\right)$ the map $J_{s}^{1} f X^{(s)} \rightarrow J_{s}^{1}\left(X, \mathbb{R}^{n}\right)$ is given by $J_{s}^{1} f\left(x_{1}, \quad, x_{s}\right)=\left(J^{1} f\left(x_{1}\right), \quad, J^{1} f\left(x_{s}\right)\right)$, where $J^{1} f(x) \in J^{1}\left(X, \mathbb{R}^{n}\right)$ is the 1 -jet generated by $f$ and $x \in X$ Denote by $V_{\omega}$ the set of those

$$
\tau=\left(J^{1} f_{1}\left(x_{1}\right), \quad, J^{1} f_{s}\left(x_{s}\right)\right) \in J_{s}^{1}\left(X, \mathbb{R}^{n}\right)
$$

such that for every $\imath \in \operatorname{Im} \omega$, rank $d f_{i}\left(x_{1}\right)=n-1$ and the vector $f_{j}\left(x_{j}\right)-f_{l}\left(x_{l}\right)$ is not tangent to $f_{t}(X)$ at the point $f_{l}\left(x_{i}\right)$ for all $j \in I_{l}(\omega)$ Consıder the open submanifold $M$ of $J_{s}^{1}\left(X, \mathbb{R}^{n}\right)$ given by

$$
M=\left(\alpha^{s}\right)^{-1}\left(X^{(s)}\right) \cap\left(\beta^{s}\right)^{-1}\left(U_{\omega}\right) \cap V_{\omega},
$$

where $\alpha \quad J^{1}\left(X, \mathbb{R}^{n}\right) \rightarrow X, \beta \quad J^{1}\left(X, \mathbb{R}^{n}\right) \rightarrow \mathbb{R}^{n}$ are defined by

$$
\alpha\left(J^{1} f(x)\right)=x, \quad \beta\left(J^{1} f(x)\right)=f(x)
$$

(36) Given $\tau \in M$ there are coordinate neighbourhoods $V_{1}$ of elements of $X$ such that $V_{\imath} \cap V_{J}=\varnothing$ for $l \neq J$ and $\tau$ belongs to

$$
\Omega=M \cap\left(\prod_{i=1}^{s} J^{1}\left(V_{\imath}, \mathbb{R}^{n}\right)\right)
$$

Take arbitrary charts $\theta_{l} \quad V_{l} \rightarrow \mathbb{R}^{n-1}$, and consider the chart

$$
\theta \Omega \rightarrow\left(\mathbb{R}^{n-1}\right)^{(s)} \times\left(\mathbb{R}^{n}\right)^{(s)} \times \mathbb{R}^{(n-1) n s},
$$

defined by

$$
\begin{gathered}
\theta\left(J^{1} f\left(x_{1}\right), \quad, J^{1} f_{s}\left(x_{s}\right)\right)=\left(u_{1}, \quad, u_{s}, v_{1}, \quad, v_{s},\left(a_{v}^{(t)}\right)\right) \\
1 \leq t \leq n, 1 \leq t \leq s, 1 \leq j \leq n-1,
\end{gathered}
$$

where for all $t=1, \quad, s, J=1, \quad, n-1, t=1, \quad, n$ we have

$$
u_{t}=\theta_{t}\left(x_{t}\right), \quad v_{t}=f_{t}\left(x_{t}\right)
$$

and

$$
a_{i j}^{(t)}=\frac{\partial\left(f_{i}^{(t)} \circ \theta_{t}^{-1}\right)}{\partial u_{t}^{(j)}}\left(u_{i}\right)
$$

Here $f_{t}=\left(f_{1}^{(1)}, \quad, f_{1}^{(n)}\right)$ and $u_{1}=\left(u_{i}^{(1)}, \quad, u_{i}^{(n-1)}\right) \in \mathbb{R}^{n-1}$

We shall write the elements $\xi$ of $\theta(\Omega)$ in the form

$$
\xi=(u, v, a)
$$

where

$$
u=\left(u_{1}, \quad, u_{s}\right) \in\left(\mathbb{R}^{n-1}\right)^{(s)}, \quad v=\left(v_{1}, \quad, v_{s}\right) \in\left(\mathbb{R}^{n}\right)^{(s)}, \quad a=\left(a_{1}^{(t)}\right) \in \mathbb{R}^{n s(n-1)}
$$




\section{Proof of theorem $A$}

We shall consider in detail the case of non-symmetric rays

Suppose $Y=f(X), f \in C_{\mathrm{emb}}^{\infty}\left(X, \mathbb{R}^{n}\right)$, and $\gamma$ is a non-symmetric periodic reflectıng ray on $Y$ with $d(\gamma)>0$ Then (cf 33 ) there exists an admissible surjection $\omega$ of type (3 1) and an element $x=\left(x_{1}, \quad, x_{s}\right) \in X^{(s)}$ such that $y_{\omega(1)}, \quad, y_{\omega(k)}, y_{\omega(k+1)}=$ $y_{\omega(1)}$ are all successive reflection points of $\gamma$ We may assume

$$
\omega(1)=1 \text {, }
$$

and

$$
r=\operatorname{card} \omega^{-1}(1)>1
$$

Let $l_{1}<l_{2}<\quad<l_{r}$, be all the elements of $\omega^{-1}(1)$ It is clear that $f^{s}(x) \in U_{\omega}$, and

$$
\operatorname{grad}_{x^{\prime}} F \circ f^{s}(x)=0
$$

for

$$
x^{\prime}=\left(x_{2}, \quad, x_{s}\right) \in X^{(s-1)}
$$

Moreover, for every $l=1, \quad, r$, if $l=\omega\left(l_{l}-1\right)$ and $j=\omega\left(l_{l}+1\right)$, then the vectors $w_{1}=\left(y_{1}-y_{1}\right) /\left\|y_{1}-y_{1}\right\|$ and $w_{2}=\left(y_{j}-y_{1}\right) /\left\|y_{j}-y_{1}\right\|$ lie in a common (twodimensional) plane with a unit normal vector $N_{1}$ to $Y=f(X)$ at the point $y_{1}$, and $w_{1}$ and $w_{2}$ make equal angles with $N_{1}$ This is equivalent to

$$
\frac{f\left(x_{1}\right)-f\left(x_{1}\right)}{\left\|f\left(x_{1}\right)-f\left(x_{1}\right)\right\|}+\frac{f\left(x_{j}\right)-f\left(x_{1}\right)}{\left\|f\left(x_{j}\right)-f\left(x_{1}\right)\right\|}=\left\langle\frac{f\left(x_{1}\right)-f\left(x_{1}\right)}{\left\|f\left(x_{1}\right)-f\left(x_{1}\right)\right\|}+\frac{f\left(x_{j}\right)-f\left(x_{1}\right)}{\left\|f\left(x_{j}\right)-f\left(x_{1}\right)\right\|}, N_{1}\right\rangle N_{1}
$$

Now fix a non-symmetric surjection (3 1) with (4 1) and (4 2), and denote by $T_{\omega}$ the set of all $f \in C_{\mathrm{emb}}^{\infty}\left(X, \mathbb{R}^{n}\right)$ such that for every $x \in X^{(s)}$ with $f^{s}(x) \in U_{\omega}$ and (43), there exists at least one $l=1,2, \quad, r$ for which (45) is not satisfied for $l=\omega\left(l_{l}-1\right)$, $J=\omega\left(l_{l}+1\right), N_{1}$ being a unit normal vector to $f(X)$ at $f\left(x_{1}\right)$ We are going to prove $T_{\omega}$ contains a residual subset of $C_{\mathrm{emb}}^{\infty}\left(X, \mathbb{R}^{n}\right)$ To this end we shall use the open submanifold $M$ of $J_{s}^{1}\left(X, \mathbb{R}^{n}\right)$, defined by (3 8)

Introduce the set $\Sigma$ of those $\tau=\left(J^{1} f_{1}\left(x_{1}\right), \quad, J^{1} f_{s}\left(x_{s}\right)\right) \in M$ so that

$$
\operatorname{grad}_{x^{\prime}} F \circ\left(f_{1} \times \quad \times f_{s}\right)(x)=0,
$$

and for any $l=1, \quad, r$ we have

$$
\frac{f_{i}\left(x_{1}\right)-f_{1}\left(x_{1}\right)}{\left\|f_{i}\left(x_{i}\right)-f_{1}\left(x_{1}\right)\right\|}+\frac{f_{l}\left(x_{j}\right)-f_{1}\left(x_{1}\right)}{\left\|f_{j}\left(x_{j}\right)-f_{1}\left(x_{1}\right)\right\|}=\left\langle\frac{f_{i}\left(x_{i}\right)-f_{1}\left(x_{1}\right)}{\left\|f_{i}\left(x_{i}\right)-f_{1}\left(x_{1}\right)\right\|}+\frac{f_{J}\left(x_{j}\right)-f_{1}\left(x_{1}\right)}{\left\|f_{j}\left(x_{j}\right)-f_{1}\left(x_{1}\right)\right\|}, N\right\rangle N,
$$

for $t=\omega\left(l_{l}-1\right), \jmath=\omega\left(l_{l}+1\right), N$ being a unit normal vector to $f_{1}(X)$ at $f_{1}\left(x_{1}\right)$ It follows from the definitions of $T_{\omega}$ and $\Sigma$ that

$$
T_{\omega}=\left\{f \in C_{\mathrm{emb}}^{\infty}\left(X, \mathbb{R}^{n}\right) j_{s}^{1} f\left(X^{(s)}\right) \cap \Sigma=\varnothing\right\}
$$

The most important result of this section is the following

LEMMA 41 There exist smooth submanıfolds $W_{m}(m=1,2, \quad)$ of $M$ with

and

$$
\text { codim } W_{m}=s(n-1)+(r-1)(n-1), \quad(m=1,2, \quad)
$$

$$
\Sigma \subset \bigcup_{m=1}^{\infty} W_{m}
$$


Proof Consider a coordinate neighbourhood $\Omega$ of the type (39) of an element of $\Sigma$ and a chart (310) on $\Omega$ defined by (311), (3 12) and (313) It is sufficient to show that $\theta(\Omega \cap \Sigma)$ is contained in a smooth submanifold of $\theta(\Omega)$ with codimension $s(n-1)+(r-1)(n-1)$

Take $l=1, \quad, r$ and set $l=\omega\left(l_{l}-1\right), j=\omega\left(l_{l}+1\right)$ For $m \leq n$ define $d_{l}^{(m)} \theta(\Omega) \rightarrow \mathbb{R}$ by

$$
d_{l}^{(m)}(\xi)=\frac{v_{1}^{(m)}-v_{1}^{(m)}}{\left\|v_{1}-v_{1}\right\|}+\frac{v_{J}^{(m)}-v_{1}^{(m)}}{\left\|v_{J}-v_{1}\right\|}-\left\langle\frac{v_{1}-v_{1}}{\left\|v_{l}-v_{1}\right\|}+\frac{v_{\jmath}-v_{1}}{\left\|v_{j}-v_{1}\right\|}, N(\xi)\right\rangle N^{(m)}(\xi)
$$

where $\xi \in \theta(\Omega)$ is given by (3 14) and (3 15), and $N(\xi)=N^{\prime} /\left\|N^{\prime}\right\|$,

$$
N^{\prime}(\xi)=\operatorname{det}\left|\begin{array}{ccc}
e_{1} & e_{2} & e_{n} \\
a_{11}^{(1)} & a_{11}^{(2)} & a_{11}^{(n)} \\
a_{12}^{(1)} & a_{12}^{(2)} & a_{12}^{(n)} \\
a_{1 n-1}^{(1)} & a_{1 n-1}^{(2)} & a_{1 n-1}^{(n)}
\end{array}\right|,
$$

$e_{p}=(0, \quad, 0,1,0, \quad, 0)$ being the $p$ th standard basis vector in $\mathbb{R}^{n}$ Consider also the maps $b_{p q} \theta(\Omega) \rightarrow \mathbb{R}(p=2, \quad, s, q=1, \quad, n-1)$, defined by

$$
b_{p q}(\xi)=\sum_{t=1}^{n} \frac{\partial F}{\partial y_{p}^{(t)}}(v) a_{p q}^{(t)}
$$

Finally, for every $m=1, \quad, n$ set

$$
O_{m}=\left\{\xi \in \theta(\Omega) \quad N^{(m)}(\xi) \neq 0\right\}
$$

Clearly, $O_{m}$ are open subsets of $\theta(\Omega)$ and $\bigcup_{m=1}^{n} O_{m}=\theta(\Omega)$ So it is sufficient to prove that for every $m, \theta(\Omega \cap \Sigma) \cap O_{m}$ is contained in a smooth submanifold of $O_{m}$ with codimension $s(n-1)+(r-1)(n-1)$

Fix $m_{0}=1, \quad, n$ and consider the map $K O_{m_{0}} \rightarrow\left(\mathbb{R}^{n-1}\right)^{s-1+r}$, given by

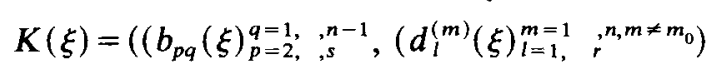

It follows from (46), (47), (4 11), (4 12) and the definition of $K$ that $O_{m_{0}} \cap$ $\theta(\Omega \cap \Sigma) \subset K^{-1}(0)$ Therefore the proof will be complete if we show that $K$ is a submersion at any point of $O_{m_{0}}$ Take $\xi \in O_{m_{0}}, \xi$ being given by $(314)$ and (3 15), and suppose

$$
\sum_{p=2}^{s} \sum_{q=1}^{n-1} B_{p q} \operatorname{grad} b_{p q}(\xi)+\sum_{l=1}^{r} \sum_{\substack{m=1 \\ m \neq m}}^{n} D_{l}^{(m)} \operatorname{grad} d_{l}^{(m)}(\xi)=0
$$

for some real numbers $B_{p q}$ and $D_{l}^{(m)}$ For fixed $p$ and $q$ by 33 there is $t=1, \quad n$ with $\left(\partial F / \partial y_{p}^{(t)}\right)(v) \neq 0$ By (414), taking into account the derivatives with respect to $a_{p q}^{(t)}$, we get $B_{p q}\left(\partial F / \partial y_{p}^{(t)}\right)(v)=0$, and therefore $B_{p q}=0$ Thus the first double sum in (4 14) is trivial Now fix $l=1, \quad, r$ and $m=1, \quad, n, m \neq m_{0}$, and set $l=\omega\left(l_{l}-1\right)$, $\jmath=\omega\left(l_{l}+1\right), N=N(\xi)$ For convenience put

$$
w=\left(v_{t}-v_{1}\right) /\left\|v_{1}-v_{1}\right\|
$$


Then by (4 14) for $t=1, \quad, n, t \neq m$, we have

$$
\frac{\partial d_{l}^{(m)}}{\partial v_{1}^{(t)}}(\xi)=\frac{1}{\left\|v_{t}-v_{1}\right\|}\left[N^{(m)} w^{(t)}\langle w, N\rangle-N^{(m)} N^{(t)}-w^{(m)} w^{(t)}\right] \quad(t \neq m)
$$

Similarly, we get

$$
\frac{\partial d_{l}^{(t)}}{\partial v_{l}^{(t)}}(\xi)=\frac{1}{\left\|v_{1}-v_{1}\right\|}\left[1+N^{(t)} w^{(t)}\langle w, N\rangle-\left(N^{(t)}\right)^{2}-\left(w^{(t)}\right)^{2}\right]
$$

Note that $\left(\partial d_{l}^{\left(m^{\prime}\right)} / \partial v_{l}^{(m)}\right)(\xi)=0$ if $l^{\prime} \neq l$, so considering in $(414)$ the derivatives with respect to $v_{t}^{(t)}$ we obtain

$$
\sum_{\substack{m=1 \\ m \neq m_{0}}}^{n} D_{l}^{(m)} \frac{\partial d_{l}^{(m)}}{\partial v_{l}^{(t)}}(\xi)=0 \quad(t=1, \quad, n)
$$

For convenience set

$$
D_{l}^{\left(m_{0}\right)}=0, \quad D_{l}=\left(D_{l}^{(1)}, \quad, D_{l}^{(n)}\right) \in \mathbb{R}^{n}
$$

Then we have

$$
\sum_{m=1}^{n} D_{l}^{(m)} \frac{\partial d_{l}^{(m)}}{\partial v_{\imath}^{(t)}}(\xi)=0 \quad(t=1, \quad, n),
$$

and (4 16) and (4 17) imply

$$
D_{l}^{(t)}+\sum_{m=1}^{n} D_{l}^{(m)}\left[N^{(m)} w^{(t)}\langle w, N\rangle-N^{(m)} N^{(t)}-w^{(m)} w^{(t)}\right]=0,
$$

that is

$$
D_{l}^{(t)}+\left\langle D_{l}, N\right\rangle\langle w, N\rangle w^{(t)}-\left\langle D_{l}, N\right\rangle N^{(t)}-\left\langle D_{l}, w\right\rangle w^{(t)}=0
$$

$(t=1, \quad, n)$, and equivalently

$$
D_{l}+\left\langle D_{l}, N\right\rangle\langle w, N\rangle w-\left\langle D_{l}, N\right\rangle N-\left\langle D_{l}, w\right\rangle w=0
$$

Taking the inner product of the left hand side of (419) with $N$ we find

$$
\left\langle D_{l}, N\right\rangle\langle w, N\rangle^{2}-\left\langle D_{l}, w\right\rangle\langle w, N\rangle=0
$$

It is not difficult to see that $\langle w, N\rangle \neq 0$ Indeed, since $\xi \in \theta(\Omega), \Omega \subset M$, we have $\xi=\theta(\tau)$ for some element $\tau$ of $M, \tau=\left(J^{1} f_{1}\left(x_{1}\right), \quad, J^{1} f_{s}\left(x_{s}\right)\right)$ Then $u, v$ and $a$ have the form (3 15) with (3 12) and (3 13) By (3 8), $M \subset V_{\omega}$, and the definition of $V_{\omega}$ 1mplies $N^{\prime}(\xi) \neq 0$ and $N(\xi)=N^{\prime}(\xi) /\left\|N^{\prime}(\xi)\right\|$ is a unit normal vector to $f_{1}(X)$ at $v_{1}=f_{1}\left(x_{1}\right)$ On the other hand, by $\imath \in I_{1}(\omega)$ and the definition of $V_{\omega}$ we have $\left\langle v_{1}-v_{1}, N(\xi)\right\rangle \neq 0$ Thus, by (415) we get $\langle w, N\rangle \neq 0$ Now (4 20) implies $\left\langle D_{l}, N\right\rangle\langle w, N\rangle=\left\langle D_{l}, w\right\rangle$, and combining this with (419) we find $D_{l}=\left\langle D_{l}, N\right\rangle N$ On the other hand, by $(418)$

$$
0=D_{l}^{\left(m_{0}\right)}=\left\langle D_{l}, N\right\rangle N^{\left(m_{0}\right)}
$$

then $\left\langle D_{l}, N\right\rangle=0$, because $N^{\left(m_{0}\right)} \neq 0$ by $(413)$ and $\xi \in O_{m_{0}}$ Hence $D_{l}=0$ which means by (4 18), $D_{l}^{(m)}=0$ for every $m=1, \quad, n$ Thus we have proved the second double sum in (4 14) is trivial which shows $K$ is a submersion in $O_{m_{0}}$ Therefore $K^{-1}(0)$ is a smooth submanifold of $O_{m_{0}}$ with codimension $(n-1)(s-1)+r(n-1)=$ $s(n-1)+(r-1)(n-1)$ 
Corollary $42 T_{\omega}$ contains a residual subset of $C_{\mathrm{emb}}^{\infty}\left(X, \mathbb{R}^{n}\right)$

Proof Since $M$ is open in $J_{s}^{1}\left(X, \mathbb{R}^{n}\right)$, any $W_{m}$ is a smooth submanifold of $J_{s}^{1}\left(X, \mathbb{R}^{n}\right)$ with the same codimension By the multijet transversality theorem ([1, ch II $]$ ) for every $m$ the set

$$
S_{m}=\left\{f \in C^{\infty}\left(X, \mathbb{R}^{n}\right) j_{s}^{1} f \text { 不 } W_{m}\right\} \cap C_{\text {emb }}^{\infty}\left(X, \mathbb{R}^{n}\right)
$$

is residual in $C_{\text {emb }}^{\infty}\left(X, \mathbb{R}^{n}\right)$ On the other hand, (4 2) and (49) imply

$$
\operatorname{cod} 1 \mathrm{~m} W_{m} \geq s(n-1)+(n-1)>s(n-1)=\operatorname{dim} X^{(s)}
$$

Since $J_{s}^{1} f X^{(s)} \rightarrow J_{s}^{1}\left(X, \mathbb{R}^{n}\right)$, we obtain

$$
S_{m}=\left\{f \in C_{\text {emb }}^{\infty}\left(X, \mathbb{R}^{n}\right) J_{s}^{1} f\left(X^{(s)}\right) \cap W_{m}=\varnothing\right\}
$$

Combinıng this with (48) and $(410)$ we get $\bigcap_{m=1}^{\infty} S_{m} \subset T_{\omega}$ which proves the corollary

Corollary 43 The set $T^{\prime}$ of those $f \in C_{\mathrm{emb}}^{\infty}\left(X, \mathbb{R}^{n}\right)$ such that every non-symmetric periodic reflecting ray on $f(X)$ has zero defect, contains a residual subset of $C_{\mathrm{emb}}^{\infty}\left(X, \mathbb{R}^{n}\right)$

Proof The assertion follows from $T^{\prime} \supset \bigcap_{\omega} T_{\omega}$, where $\omega$ runs over the non-symmetric surjections (3 1) with (4 1) and (4 2)

In a similar way one can deal with the symmetric rays We shall point out only a few differences Suppose $\gamma$ is a symmetric periodic reflectıng ray on $Y=f(X)$ Then we can find a symmetric surjection (3 1) such that $\gamma$ is of type $\omega$ By the definition of symmetric map we have $k=2 m$, moreover we can choose $\omega$ in such a way that (3 3) is fulfilled for $t_{0}=1$ Suppose $d(\gamma)>0$ Then for some $J_{0}=1, \quad, s$ we have

$$
r=\operatorname{card}\left(\omega^{-1}\left(J_{0}\right) \cap\{1,2, \quad, m+1\}\right)>1
$$

Let $l_{1}<l_{2}<\quad<t_{r}$ be the elements of $\omega^{-1}\left(J_{0}\right) \cap\{1, \quad, m+1\}$ Given $x=$ $\left(x_{1}, \quad, x_{s}\right) \in X^{(s)}$, define $x^{\prime}$ by $x^{\prime}=\left(x_{1}, \quad, x_{j_{0}-1}, x_{j_{0}+1}, \quad, x_{s}\right)$ Further, we go on as in the non-symmetric case, proving that for every symmetric surjection $\omega$ with the properties listed above (including (4 21) for some $J_{0}=1, \quad, s$ ) the set $T_{\omega}$ contains a residual subset of $C_{\mathrm{emb}}^{\infty}\left(X, \mathbb{R}^{n}\right)$ Thus we establish the set $T^{\prime \prime}$ of those $f \in$ $C_{\mathrm{emb}}^{\infty}\left(X, \mathbb{R}^{n}\right)$ so that every symmetric periodic reflectıng ray on $f(X)$ has zero defect, contains a residual subset of $C_{\mathrm{emb}}^{\infty}\left(X, \mathbb{R}^{n}\right)$

To conclude the proof of theorem $A$, we mention that $T^{\prime} \cap T^{\prime \prime} \subset \mathscr{A}$ Therefore $\mathscr{A}$ also contains a residual subset of $C_{\mathrm{emb}}^{\infty}\left(X, \mathbb{R}^{n}\right)$

\section{Proof of theorem $B$}

Suppose $\gamma_{1}$ and $\gamma_{2}$ are two different non-symmetric periodic reflectıng rays on $Y=f(X), f \in C_{\mathrm{emb}}^{\infty}\left(X, \mathbb{R}^{n}\right)$ Let $y_{1}, \quad, y_{s}$ be all different reflection points of $\gamma_{1}$ and $\gamma_{2}$ taken together, $y_{1}=f\left(x_{1}\right), x_{1} \in X, t=1, \quad, s$ Then there exist admissible nonsymmetric maps

$$
\omega\{1, \quad, k\} \rightarrow\{1, \quad, s\}, \quad \delta\{1, \quad, l\} \rightarrow\{1, \quad, s\}
$$

with

$$
\operatorname{Im} \omega \cup \operatorname{Im} \delta=\{1, \quad, s\}
$$


and

$$
\{\omega(l), \omega(l+1)\} \neq\{\delta(J), \delta(j+1)\} \quad(1 \leq l \leq k, 1 \leq J \leq l)
$$

such that $\gamma_{1}$ is of type $\omega$ and $\gamma_{2}$ is of type $\delta$ (cf 33 ) Note especially that $\left(\begin{array}{ll}5 & 2\end{array}\right)$ expresses the fact that $\gamma_{1}$ and $\gamma_{2}$, being different, have no common segments

As in [9], the symbol $\Gamma=(k, l, s, \omega, \delta)$ with the above properties will be called a configuration, and the pair of rays $\left(\gamma_{1}, \gamma_{2}\right)$ will be said to be of type $\Gamma$ In this case we have $y=\left(y_{1}, \quad, y_{s}\right) \in U_{\omega} \cap U_{\delta}$ and the periods (lengths) of $\gamma_{1}$ and $\gamma_{2}$ coincide with $F(y)$ and $G(y)$, respectively, where $F, G\left(\mathbb{R}^{n}\right)^{(s)} \rightarrow \mathbb{R}$ are given by

$$
\begin{aligned}
& F\left(z_{1}, \quad, z_{s}\right)=\sum_{i=1}^{h}\left\|z_{\omega(t)}-z_{\omega(t+1)}\right\|, \\
& G\left(z_{1}, \quad, z_{s}\right)=\sum_{t=1}^{l}\left\|z_{\delta(t)}-z_{\delta(t+1)}\right\|
\end{aligned}
$$

As for $\omega$, set $\delta(p)=\delta(l)$ if $p=l+m l, 1 \leq l \leq l, m$ an integer

Suppose $\gamma_{1}$ and $\gamma_{2}$ have at least one common reflection point Without loss of generality we may assume $y_{1}$ is a reflection point for both $\gamma_{1}$ and $\gamma_{2}$, that is $1 \in \operatorname{Im} \omega \cap \operatorname{Im} \delta$ Moreover, we may assume

$$
\omega(1)=\delta(1)=1
$$

Set

$$
\iota^{\prime}=\omega(k), \quad J^{\prime}=\omega(2), \quad \iota^{\prime \prime}=\delta(l), \quad J^{\prime \prime}=\delta(2)
$$

Then (4 5) is satisfied for $\imath=l^{\prime}, J=J^{\prime}$ and for $\imath=l^{\prime \prime}, J=J^{\prime \prime}$, where $x=\left(x_{1}, \quad, x_{s}\right) \in X^{(s)}$ and $N_{1}$ is a unit normal vector to $Y=f(X)$ at $y_{1}$ Introduce the function

$$
H\left(\mathbb{R}^{n}\right)^{(s)} \rightarrow \mathbb{R}
$$

defined by $H(z)=F(z)+G(z)$ Since $x$ is a critical point for both $F \circ f^{s}$ and $G \circ f^{s}$, it is a critical point for $H \circ f^{s}$ too In particular,

$$
\operatorname{grad}_{x} H \circ f^{s}(x)=0 \text {, }
$$

where $x^{\prime}$ is defined by $(44)$

We are going to use the argument from the proof of theorem $A$, replacing $F$ by $H$ To do this we need a property of $H$ similar to 34 In fact, what we need is equivalent to

$$
\sum_{J \in I_{i}(\omega)} \frac{y_{1}-y_{J}}{\left\|y_{1}-y_{j}\right\|}+\sum_{J \in I_{1}(\delta)} \frac{y_{1}-y_{j}}{\left\|y_{1}-y_{j}\right\|} \neq 0
$$

for $l=2, \quad, s, l \in \operatorname{Im} \omega \cap \operatorname{Im} \delta$ and $y=\left(y_{1}, \quad, y_{s}\right) \in U_{\omega} \cap U_{\delta}$ However, this is not true in general (see for example figure 2) That is why we have to make a little modification of the argument from $\S 4$

Denote by $\mathscr{B}_{\Gamma}$ the set of those $f \in C_{\mathrm{emb}}^{\infty}\left(X, \mathbb{R}^{n}\right)$ such that if $x \in X^{(s)}$ is a critical point of $F \circ f^{s}$ with $f^{s}(x) \in U_{\omega} \cap U_{\delta}$ and (57), then (4 5) is not satisfied either for $\imath=t^{\prime}$ and $j=\jmath^{\prime}$ or for $\imath=t^{\prime \prime}$ and $\jmath=\jmath^{\prime \prime}$ It follows from above that

$$
\bigcap_{\Gamma} \mathscr{B}_{\Gamma} \subset \mathscr{B} \text {, }
$$

where $\mathscr{B}$ is the set defined in theorem $\mathrm{B}$, and $\Gamma=(k, l, s, \omega, \delta)$ runs over those configurations with (5 5) 
Fix a configuration $\Gamma=(k, l, s, \omega, \delta)$ with (5 5) and non-symmetric $\omega$ and $\delta$, and define $F, G, H, \iota^{\prime}, \jmath^{\prime}, \iota^{\prime \prime}, \jmath^{\prime \prime}$ as above We have to show $\mathscr{B}_{\Gamma}$ contains a residual subset of $C_{\mathrm{emb}}^{\infty}\left(X, \mathbb{R}^{n}\right)$ For $\iota=1, \quad, s$ define

$$
u_{\iota}=\left\{y \in U_{\omega} \cap U_{\delta} \operatorname{grad}_{y_{1}} H(y) \neq 0\right\}
$$

and set $\tilde{u}=\bigcap_{t=1}^{s} U_{t}$ All $U_{t}$ are open subsets of $U_{\omega} \cap U_{\delta}$ (therefore $\tilde{U}_{1}$ also open), and $U_{\imath}=U_{\omega} \cap U_{\delta}$ if $\imath \notin \operatorname{Im} \omega \cap \operatorname{Im} \delta$ ( $\operatorname{cf} 34$ )

Denote by $T_{\Gamma}$ the set of those $f \in C_{\mathrm{emb}}^{\infty}\left(X, \mathbb{R}^{n}\right) y$ such that for every $x \in X^{(s)}$ with $f^{s}(x) \in \tilde{U}$ and (57), (45) is not fulfilled either for $t=\imath^{\prime}, j=\jmath^{\prime}$ or for $\imath=\imath^{\prime \prime}, \jmath=\jmath^{\prime \prime}$

LEMMA $51 T_{\Gamma}$ contains a residual subset of $C_{\mathrm{emb}}^{\infty}\left(X, \mathbb{R}^{n}\right)$

The proof uses the same argument as those used in $\S 4$ to show $T_{\omega}$ contains a residual subset of $C_{\mathrm{emb}}^{\infty}\left(X, \mathbb{R}^{n}\right)$

Further, for $\imath \in \operatorname{Im} \omega \cap \operatorname{Im} \delta$ let $T$, be the set of those $f$ in $C_{\mathrm{emb}}^{\infty}\left(X, \mathbb{R}^{n}\right)$ such that if $x \in X^{(s)}$ is a critical point of $F \circ f^{s}$ and $f^{s}(x) \in U_{\omega} \cap U_{\delta}$, then $f^{s}(x) \in \mathcal{U}_{\text {, We }}$ mention that

$$
\bigcap_{i \in \operatorname{Im} \omega \cap \operatorname{Im} \delta} T_{\imath} \cap T_{\Gamma} \subset \mathscr{B}_{\Gamma}
$$

Indeed, suppose $f$ belongs to the left hand side of (58) and $x \in X^{(s)}$ is a critical point of $F \circ f^{s}$ with $f^{s}(x) \in U_{\omega} \cap U_{\delta}$ and (57) Then for every $\imath \in \operatorname{Im} \omega \cap \operatorname{Im} \delta, f \in T_{\imath}$ implies $f^{s}(x) \in \mathscr{U}$ Thus $f^{s}(x) \in \tilde{U}$, and now by $f \in T_{\Gamma}$ we see that (45) is not satisfied either for $\imath=\iota^{\prime}, \jmath=\jmath^{\prime}$ or for $\imath=\iota^{\prime \prime}, \jmath=\jmath^{\prime \prime}$ Therefore $f \in \mathscr{B}_{\Gamma}$

Fix $\imath \in \operatorname{Im} \omega \cap \operatorname{Im} \delta$ To prove that $T$, contains a residual subset of $C_{\mathrm{emb}}^{\infty}\left(X, \mathbb{R}^{n}\right)$ we shall use the following result which is a part of theorem $31 \mathrm{in} \mathrm{[9]}$

THEOREM $52([9])$ Let $n \geq 2, s \geq 2, q \geq 1$ be integers, $U$ be an open subset of $\left(\mathbb{R}^{n}\right)^{(s)}$, $H \quad U \rightarrow \mathbb{R}$ and $L \quad U \rightarrow \mathbb{R}^{q}$ be smooth maps Suppose $L$ has no crittcal points in $U$ and $\operatorname{grad}_{y_{1}} H(y) \neq 0$ for all $l=1, \quad, s$ and $y \in U$ Let $X$ be a smooth $(n-1)$-dimensional submanifold of $\mathbb{R}^{n}$ and $T$ be the set of those $f \in C_{\mathrm{emb}}^{\infty}\left(X, \mathbb{R}^{n}\right)$ such that for every $x \in X^{(s)}$ with $f^{s}(x) \in U$ which is a critical point of $H \circ f^{s}$, we have $L\left(f^{s}(x)\right) \neq 0$ Then $T$ contains a residual subset of $C_{\mathrm{emb}}^{\infty}\left(X, \mathbb{R}^{n}\right)$

To apply the theorem define $L\left(\mathbb{R}^{n}\right)^{(s)} \rightarrow \mathbb{R}^{n}$ by

$$
L^{(t)}(y)=\frac{\partial F}{\partial y_{\imath}^{(t)}}(y)+\frac{\partial G}{\partial y_{\imath}^{(t)}}(y) \quad(t=1, \quad, n),
$$

and $L=\left(L^{(1)}, \quad, L^{(n)}\right)$ The following property of $L$ (established in [9]) can easily be proved by direct computations

LEMMA $53([9]) \quad D L(y) \neq 0$ for every $y \in\left(\mathbb{R}^{n}\right)^{(s)}$

Now, applying theorem 52 for $H, U=U_{\omega} \cap U_{\delta}$ and $L$, we deduce that $T_{1}$ contains a residual subset of $C_{\mathrm{emb}}^{\infty}\left(X, \mathbb{R}^{n}\right)$ Hence $\mathscr{B}_{\Gamma}$ also contaıns a residual subset of $C_{\text {emb }}^{\infty}\left(X, \mathbb{R}^{n}\right)$

In case both $\omega$ and $\delta$ are symmetric or one of them is symmetric and the other is non-symmetric, we use the same arguments with minor changes to see that $\mathscr{B}_{\Gamma}$ contains a residual subset of $C_{\mathrm{emb}}^{\infty}\left(X, \mathbb{R}^{n}\right)$ We omit the details 


\section{REFERENCES}

[1] M Golubitsky \& V Guillemın Stable Mappings and their Singularitıes Springer New York, 1973

[2] V Guillemin \& $\mathrm{R}$ Melrose The Poisson summation formula for manifolds with boundary $A d v$ in Math 38 (1979), 204-233

[3] M Hirsch Differential Topology Springer New York, 1976

[4] M Kac Can one hear the shape of a drum? Amer Math Soc Monthly 73 (1966), 1-23

[5] V Lazutkın Convex Billıards and Eigenfunctions of the Laplace Operator Ed Lenıngrad University, 1981 (in Russian)

[6] S Marvızı \& R Melrose Spectral invariants of convex planar regions $J$ Diff Geometry 17 (1982), 475-502

[7] V Petkov Poisson relation for manifolds with boundary Svmpostum and Workshop on Hyperbolic Equations and Related Topics Kyoto, 1984, 317-327

[8] V Petkov Propriétes génériques des rayons refleckıssants et applicatıons aux problèmes spectraux Semınatre Bony-Sjostrand-Mever, Ecole Polytechnıque, Centre de Mathémattques, Expose XII (19841985)

[9] V Petkov \& L Stojanov Perıods of multıple reflectıng geodesıcs and inverse spectral results Amer J Math 109 (1987)

[10] $\mathrm{V}$ Petkov \& L Stojanov Periodic geodesics of generic non-convex domains in $\mathbb{R}^{2}$ Notas $e$ Comunicaçoes de Matematıca, Universıdade Federal de Pernambuco Recıfe-Brasıl, No 138 (1985)

[11] V Petkov \& L Stojanov Spectrum of the Poincaré map for perıdıc reflectıng rays in generic domains Math $Z 194$ (1987), 505-517 